\title{
Radiation Dose Management in Pediatric CT
}

\author{
Francis R. Verdun • Leonor Alamo • \\ Frédéric A. Miéville • François Gudinchet
}

Published online: 28 December 2012

(c) Springer Science+Business Media New York 2012

\begin{abstract}
The rapid increase in the number of computed tomography (CT) scans performed has augmented concerns about safety risks. Although some of this increase is certainly legitimate and improves patient care, there is certainly still a need for dose reduction. Patients may insist on receiving a CT scan, and defensive medicine can certainly be a source of useless exposure. This means that the principle of justifying $\mathrm{CT}$ examinations needs to be reemphasized. Dealing with a properly justified examination is then a matter of image optimization aimed at providing the necessary diagnostic information at the lowest possible applied dose. The optimization of $\mathrm{CT}$ acquisition protocols involves teamwork in which manufacturers, legal bodies, radiologists, technologists, and medical physicists all play an important role. The purpose of this article is to review the latest progress in terms of the optimization of $\mathrm{CT}$ acquisition protocols in the pediatric population.
\end{abstract}

Keywords Pediatric CT - Radiation dose reduction Patient dose optimization - Radiation - Exposure

\section{Introduction}

Diagnostic X-rays contribute about $25 \%$ of the total (manmade plus natural) annual amount of radiation delivered to

\footnotetext{
F. R. Verdun $(\bowtie) \cdot$ F. A. Miéville

Institute of Radiation Physics, University Hospital Center and University of Lausanne, Rue du Grand-Pré 1,

1007 Lausanne, Switzerland

e-mail: francis.verdun@chuv.ch

L. Alamo $\cdot$ F. Gudinchet

Department of Radiology, University Hospital Center

and University of Lausanne, Lausanne, Switzerland
}

the general population in Western countries, with computed tomography (CT) being the largest single source of these $\mathrm{X}$-rays. Most patients certainly benefit from these CT examinations, but in the last 10 years their impact on the collective dose has drastically increased and, therefore, efforts are required to control this trend and to ensure that the risk-benefit ratio clearly lies on the benefit side [1-3]. The most efficient way to improve protection of the population from radiation in the field of medical imaging is, obviously, to focus on justifying examinations. This is a prerequisite to comply with regulations and certainly, at the moment, there is still a lack of consensus among certain indications for CT. Every effort should be made to optimize the acquisition protocols to avoid any waste of radiation dose. Within the process of optimization, it is essential that the dose reduction schemes proposed are verified to remain compatible over time with the production of images in which the integrity of the required diagnostic information is achieved.

Since its introduction, CT has been subject to major technological progress and modern CT units provide both a short acquisition time and an excellent isotropic 3D spatial resolution. This has drastically extended the role of CT for a broad range of clinical symptoms. The use of CT in adults has increased drastically in this last decade, and its continuous technological progress has also allowed an improvement in children's care. It is particularly useful for diagnosis and assessment of congenital heart abnormalities, lung and airway diseases, trauma, and infections [4-9] and the proportion of pediatric patients undergoing CT scans ranges from 0 to $38 \%$, with more than $10 \%$ in the USA, depending on the country and examination [10••, 11-13]. $\mathrm{CT}$ examinations can be performed in very young children, as shown by a Swiss study which demonstrated that $41 \%$ of pediatric CT examinations were performed in neonates and children under the age of 5 years [14]. 
The risk of induction of cancer from diagnostic X-ray exposure is still widely debated. In general, two main approaches are taken [15]. On the one hand, some scientists publish data predictions about the number of potential deaths following diagnostic examinations [16-19]. These data are then reformulated and simplified, reinforcing the impression that CT examinations are particularly dangerous. To achieve more impact, this kind of sensationalist literature does not mention the benefit associated with CT examinations. The other approach is to consider, as Durand [20] did in a recently, that "cumulated dose estimates are of little relevance and never constitute a logical reason to avoid an imaging procedure that is otherwise medically indicated." This last statement is certainly true for well-justified examinations, but one should never ignore the risk associated with the use of X-rays. To reinforce that statement, it is interesting to consider the results of a recent retrospective cohort study performed in the UK between 1985 and 2002 that considered CT performed on patients younger than 22 years. This study showed that cumulative doses of about $50 \mathrm{mGy}$ might almost triple the risk of leukemia and doses of about $60 \mathrm{mGy}$ might triple the risk of brain cancer. Although the cumulative absolute risks are indeed very small (one excess case of leukemia and one excess case of brain tumor per 10,000 head CT scans is estimated to occur $[21,22 \bullet \bullet])$, these results prove that every effort should be devoted to optimizing as much as possible the imaging protocols and to limit additional examinations to those that are only strictly necessary. Fortunately, many CT examinations deliver doses well below organ doses of $50 \mathrm{mGy}$ but still, the linear no-threshold model should be used to optimize CT acquisition protocols even if we know that the statistical power of the studies is not strong enough to demonstrate a link between the dose and induction of cancer.

\section{Patient Dose Optimization: A Teamwork}

In parallel to the race to increase the temporal and spatial resolutions in $\mathrm{CT}$, the optimization of the acquisition protocols has been a priority for many years, and very efficient results have already been obtained. To limit any waste of dose while maintaining the diagnostic value of the images, it is necessary to involve several actors.

\section{Role of Manufacturers}

Several innovations have been introduced over the last few years by equipment manufacturers. One relevant innovation was the introduction of tube current modulation (TCM), available as long as 10 years ago, that made it possible to limit patient overexposure. Furthermore, and compared with an already optimized protocol, TCM makes it possible to decrease the X-ray tube charge (mAs) by roughly $20 \%$ in adults [23]. Unfortunately, TCM in neonates and young children usually leads to a much more moderate dose reduction (from 2 to $10 \%$ ) when compared with already well optimized CT protocols [24].

Moreover, both the X-ray collimation and the number of detector rows have been increased over these last few years in order to increase the temporal resolution in an attempt to provide an excellent isotropic spatial resolution This trend has reduced the overbeaming effect (penumbra), but nowadays the use of large X-ray beam collimation introduces a new challenge concerning z-overscanning [25]. Fortunately, the introduction of dynamic X-ray beam collimation makes it possible to reduce this type of overexposure [26-29].

Temporal resolution can be increased significantly by the use of dual-source CT units (DSCT), where high pitch values can be used. As shown by Lell et al. [30], the use of high pitch values during chest imaging can increase the image quality in infants and small or uncooperative children without the need for sedation or controlled ventilation. In addition, the use of DSCT units makes possible the use of prospective ECG-gated sequential acquisition in cardiac $\mathrm{CT}$ in children, even in the case of heart rates over 100 beats per minute for a dose burden comparable to that of cardiac CT with nongated acquisition [31••].

One of the latest technological innovations aims to reduce the exposure of particularly radiosensitive organs such as the thyroid in children, the breast gland, and the eye lens. To achieve this goal, the X-ray tube current is modulated in the $x, y$, and $z$ directions, with a drastic tube current reduction when the tube is positioned above the organs to be spared. To produce an acceptable image quality, the introduction of this technology is associated with a compensatory organ dose increase posteriorly [32-34].

Finally, major efforts have been dedicated to optimizing the use of acquired information by replacing the standard filtered back projection (FBP) algorithm by some iterative solutions. In 2009, GE Healthcare (Milwaukee, WI, USA) introduced the first CT iterative reconstruction method commercially available for clinical applications, under the name of adaptive statistical iterative reconstruction $\left(\mathrm{ASiR}^{\mathrm{TM}}\right)$. ASIR uses a blend of FBP images with iteratively reconstructed images that are produced, according to the manufacturer, in the raw data domain to reduce the image noise [35, 36•, 37]. The same year, Siemens (Erlangen, Germany) released an iterative reconstruction solution in image space (IRIS ${ }^{\mathrm{TM}}$ ). In contrast to ASiR, IRIS is based on an iterative reconstruction loop into the image domain to speed up the reconstruction process [38, 39]. One year later, Philips commercialized the fourth version of iterative reconstruction, which was named iDose ${ }^{4 T M}$. This method attempts to reduce the image noise without introducing a 
modification of the noise power spectrum to limit the noise texture modification [38-40]. After noise removal in the raw data domain, an optimal anatomical model is used in the image domain to maintain the appearance of the full-dose image. Toshiba (Tochigi, Japan) has also developed an iterative method, adaptive iterative dose reduction $\left(\mathrm{AIDR}^{\mathrm{TM}}\right.$ ), which is adaptive and automatically calculates the optimized number of iterations [41]. Although these different methods of CT image reconstruction are still extensively evaluated, it appears that they are compatible with clinical requirements in terms of computing time, allowing a further dose reduction of 30-40\% from already optimized solutions with FBP [42-45]. Nevertheless, there is still a lot of work to be done to improve the iterative reconstruction solutions available, namely, in terms of producing acquisitions associated with an effective dose lower than $1 \mathrm{mSv}$ [46-48]. Figure 1 shows the potential for dose reduction with FBP, ASiR, and Veo (GE Healthcare). Depending on the complexity of the iterative algorithms, the image quality is highly improved. However, it has been shown that with certain of these iterative solutions, the potential for dose reduction was different depending on the reconstruction planes and the modification of the image texture (Figs. 2, 3) [48].

The challenge is now to define the indications for which such low-dose acquisitions are suited. This represents the ultimate optimization process - to associate the dose burden with the diagnostic question to be answered.

\section{Role of Legal Bodies and Professional Societies}

There is no dose limit to be applied for patients since they directly benefit from a justified radiological examination. However, in order to limit the spread of this practice, the concept of the diagnostic reference level (DRL) was introduced by the International Commission on Radiological Protection in 1997 [49]. Several studies have shown that this initiative has been very efficient in reducing the range of doses that have been applied especially to the pediatric population, which constitutes a group not very often examined with CT in small radiology centers [50]. For example, the Swiss Federal Office for Public Health has published a set of DRLs that are used during the legal auditing of the centers performing CT examinations [51]. Unfortunately, the availability of DRLs does not prevent large dose variations (up to 32-fold), as shown in a recent review that collected information regarding effective dose levels during the commonest CT examinations in adults and children [52]. However, there are very efficient initiatives such as the "image gently" campaign [53], organized under the auspice of the Society of Pediatric Radiology, and Image Wisely [54] that should help avoid an unacceptable spread of the actual practice.

\section{Role of Radiologists and Technologists}

\section{Choice of the Image Quality and Number of Acquisition Phases}

Having a framework to assess the standard protocols to be used is the first step of optimization. Publications are available to help radiologists verify whether their protocols are compatible with those commonly applied. However, the image quality should be adapted to the required diagnostic question. This will be more and more the case with the general use of iterative reconstructions. These reconstructions may allow massive dose reductions but the conspicuity of some anatomical details might be impaired. However very low dose protocols might fully compatible with follow-up studies of oncologic patients during treatment or follow-up of chronic diseases such as cystic fibrosis. The radiologist should help the technologist set the image quality. Moreover, the use of multiphase CT protocols, as those often performed for follow-up in oncologic adult patients, is rarely helpful for pediatric body scanning and should be eliminated whenever possible.
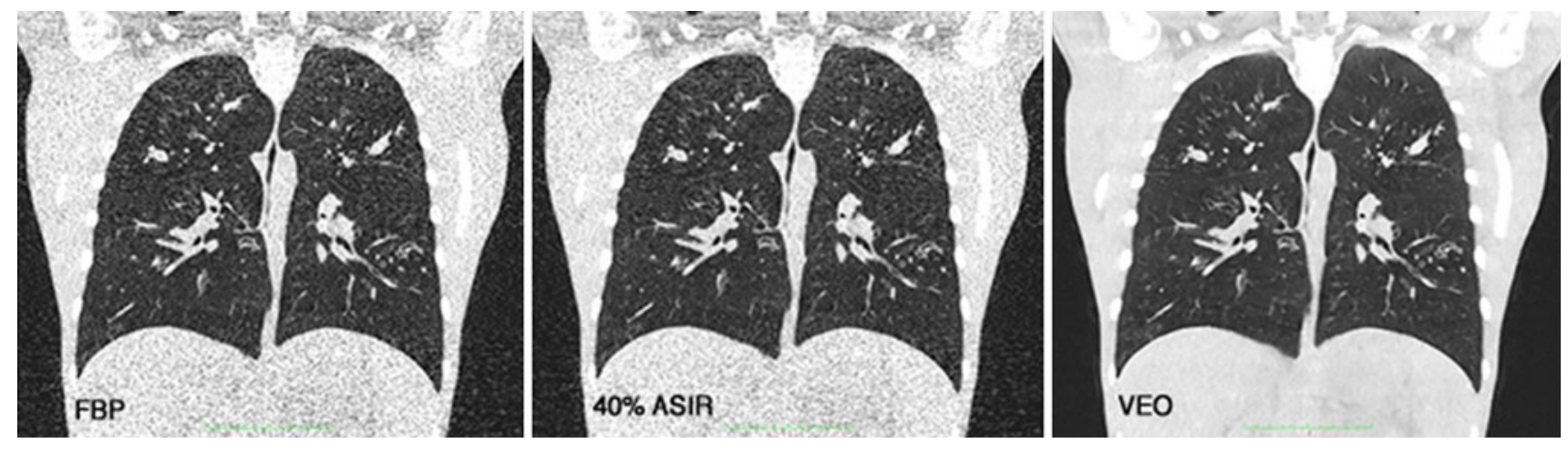

Fig. 1 Comparison of the coronal thoracic images obtained with filtered back projection $(F B P), 40 \%$ ASiR, and Veo in a 15-year-old child with a volume CT dose index $\left(\mathrm{CTDI}_{\mathrm{vol}}\right)$ of $1.5 \mathrm{mGy}$ 
Fig. 2 Comparison of the axial thoracic images obtained with FBP and Veo in an 8-year-old child with a CTDI $\mathrm{vol}_{\mathrm{vo}}$ of 0.14 mGy $(100 \mathrm{kVp}, 4 \mathrm{mAs}$, dose-length product of $3.82 \mathrm{mGy} \mathrm{cm)}$
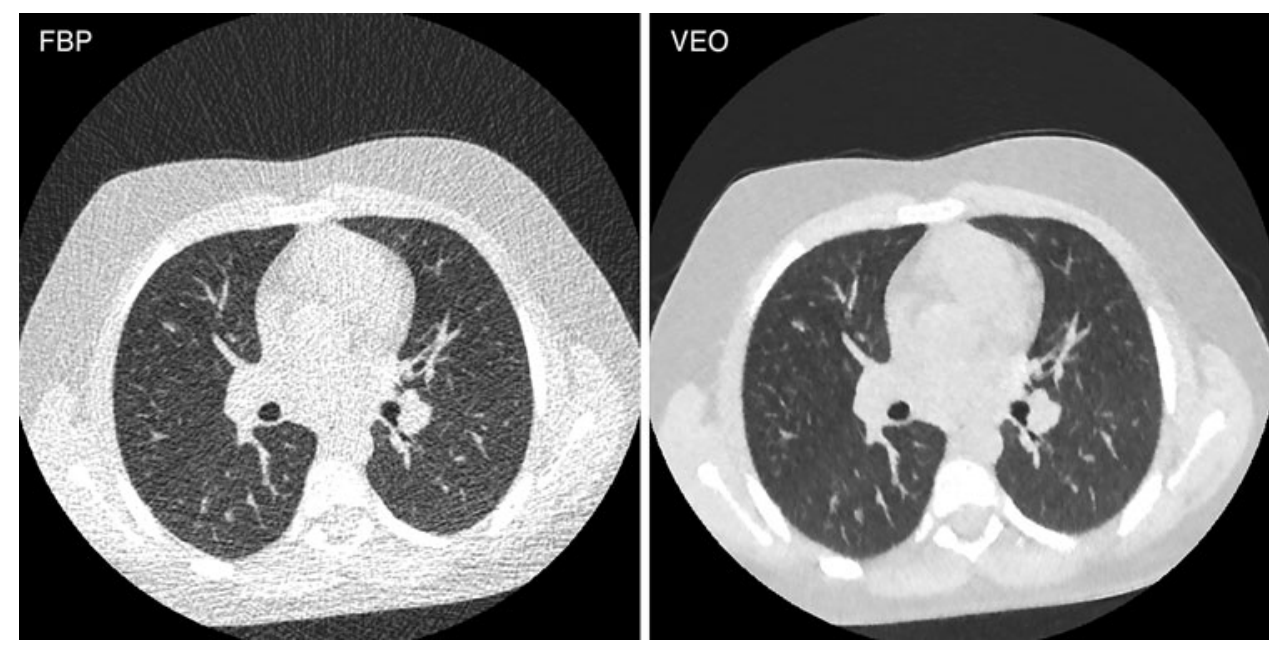

Fig. 3 Comparison of the coronal thoracic images reconstructed with FBP and Veo for the patient in Fig. 2

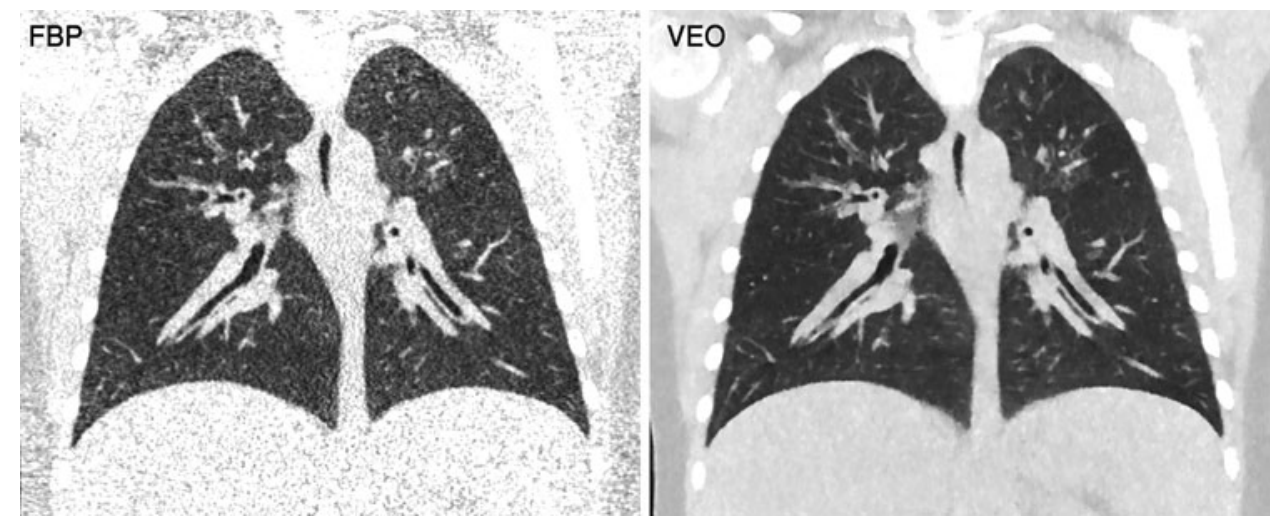

\section{Choice of the X-Ray Beam Energy}

The choice of X-ray beam energy should depend on the indication. The use of low kilovoltages does not reduce the patient dose per se, which should be controlled by the displayed dose indicators-volume CT dose index $\left(\mathrm{CTDI}_{\mathrm{vol}}\right)$ and dose-length product (DLP). However, the use of lower kilovoltages increases the photoelectric effect in bone and in tissues opacified with iodine. This increase in contrast obtained generates better conspicuity even if the image noise is increased. It is thus possible to reduce the $\mathrm{CTDII}_{\mathrm{vol}}$ without impairing the detectability of bone detail or small vessels $[55,56]$.

\section{Choice of the Pitch Value}

The pitch parameter should be used not to reduce the patient dose but to optimize the temporal resolution of the acquisition. To ensure good data sampling in the $z$-direction, one should choose a pitch value close to unity. This might lead to artifacts because of patient movement, but then the acquisition time can be reduced by increasing the pitch value. On DSCT units it is possible to use a pitch value above 3.0, making it possible to avoid patient sedation in some cases.

\section{Adaptation of the Protocol to Patient Anatomy}

The variation of noise as a function of the $\mathrm{CTDI}_{\mathrm{vol}}$ parameter significantly depends on the morphology of the patient [57]. The smaller the patient, the lower the effect of a CTDI ${ }_{\text {vol }}$ increase on noise reduction. One of the most efficient ways to control the image noise level while ensuring adequate exposure of the patient is to use the automatic exposure control that controls the TCM [13].

\section{Patient Positioning}

The patient should always be correctly centered in the CT gantry. The centering of the patient decreases the number of artifacts and increases the distance from the radiation source, therefore reducing the dose. Moreover, bow-tie filters have been designed for a properly centered patient. If the patient is not correctly centered, the inappropriate compensation of the X-ray beam by the bow-tie filter will allow more $\mathrm{X}$-rays to penetrate thinner peripheral portions 
of the patient's body, and the X-rays needed to penetrate the thicker, central portions will be reduced in intensity $[13,58]$.

\section{Organ Shielding}

Selective organ shielding was introduced 15 years ago. It consists of placing latex sheets containing bismuth on the organs to be protected: usually the breast, the thyroid, and the eye lens. Different studies have proved their efficacy in reducing the dose to the organ under which the shield was placed. However, these shields also generate a waste of dose as described by Geleijns et al. [59•]. In fact, their use is considered counterproductive, especially for the pediatric population. Currently, the best solution to use to spare organ dose is to reduce the $\mathrm{CTDI}_{\mathrm{vol}}$ as much as possible, and take advantage of the organ-based TCM that should soon be available on CT units [32].

\section{Child-Friendly Environment}

It is important to take care of the environment where the $\mathrm{CT}$ is performed, and the staff should make every effort to minimize the anxiety of the child. It is also important to reduce the time when the child is on the CT table. Distraction devices (music, toys, flashing lights, child-friendly images, etc.) may also help to lower the stress associated with a CT examination [13].

\section{Role of Medical Physicists}

The role of medical physicists should be devoted to ensuring that all the possibilities available on the CT unit to optimize patient exposure are correctly used. They should also verify the indication of the dose indicators $\left(\mathrm{CTDI}_{\mathrm{vol}}\right.$, DLP, and dose efficiency parameters) and check if the standard protocols are compatible with the recommended DRL. They should also promote staff coaching in order to increase awareness levels in terms of the dose problematic [60, 61]. Medical physicists should clearly explain the impact of the size of the patient on his/her exposure. The $\mathrm{CTDI}_{\mathrm{vol}}$ is certainly a very convenient tool to characterize CT protocols, but it does not correspond to the actual dose delivered to a given patient. In very young children, it drastically underestimates the dose delivered to the tissues. The conversion of DLP into effective dose is also a problem where medical physicists should be active [62]. The first thing to remember is that the concept of effective dose was developed for the protection of workers exposed to radiation. However, in the field of medicine it is a rather convenient tool to compare various radiological procedures, but it should not be applied on an individual basis. For pediatric patients, specific conversion factors should be used. These conversion factors should be specified separately for both sexes and take into account the X-ray spectrum used.

\section{Conclusion}

The increasing number of CT scans performed on pediatric patients raises concerns about the risks associated with radiation. Obviously, the most efficient way to improve radiation protection is to strengthen the principle of justifying CT examinations. In recent years, however, different technical advances aimed at optimizing imaging protocols and reducing applied doses have been developed. These advances make it possible to increase the temporal and/or spatial resolution (increase of the number of detector rows, DSCT with the possibility of high pitch values) as well as to reduce the required dose (iterative reconstruction methods) while maintaining image quality sufficient for diagnostic purposes.

Optimization of pediatric CT studies remains a task in which all the different entities involved (manufacturers, pediatric physicians, radiologists, technologists, medical physicists, and professional societies) play a significant role. Appropriate knowledge of radiation dose management is essential for improving the benefits and reducing the risks of pediatric CT examinations.

Acknowledgments This research project was partially funded by the Swiss National Science Foundation.

Disclosure No potential conflicts of interest relevant to this article were reported.

\section{References}

Papers of particular interest, published recently, have been highlighted as:

- Of importance

•- Of major importance

1. National Council on Radiation Protection and Measurements, "Ionizing radiation exposure of the population of the United States," NCRP report no. 160. Bethesda, 2009.

2. Etard C, Sinno-Tellier S, Aubert B. Exposure of French population by ionizing radiation due to medical diagnostic examinations in 2007. Joint report of the Institut de Radioprotection et de Sûreté Nucléaire and the Institut de Veille Sanitaire. [IRSN-IVS 2010] Paris 2010.

3. Samara ET, Aroua A, Bochud FO, Ott B, Theiler T, Treier R, Trueb PR, Vader JP, Verdun FR. Exposure of the Swiss population by medical X-rays: 2008 review. Health Phys J. 2012; 102(3):263-70.

4. Paul JF, Rohnean A, Sigal-Cinqualbre A. Multidetector CT for congenital heart patients: what a paediatric radiologist should know. Pediatr Radiol. 2010;40(6):869-75. 
5. Frush DP, Donnelly LF, Rosen NS. Computed tomography and radiation risks: what pediatric health care providers should know. Pediatrics. 2003;112(4):951-7.

6. Frush DP. Pediatric abdominal CT angiography. Pediatr Radiol. 2008;38(Suppl 2):S259-66.

7. Frush DP, Donnelly LF. Helical CT in children: technical considerations and body applications. Radiology. 1998;209(1): $37-48$.

8. Garcia-Pena P, Lucaya J. HRCT in children: technique and indications. Eur Radiol. 2004;14(Suppl 4):L13-30.

9. Papaioannou G, Young C, Owens CM. Multidetector row CT for imaging the paediatric tracheobronchial tree. Pediatr Radiol. 2007;37(6):515-29.

10. $\bullet$ Dougenia E, Faulkner K, Panayiotakisc G. A review of patient dose and optimisation methods in adult and paediatric CT scanning. Eur J Radiol. 2012; 81:e665-e683. This contribution is an excellent review and provides many data regarding the exposure of the patient.

11. United Nations Scientific Committee on the Effects of Atomic Radiation. Sources and effects of ionizing radiation. Report to the General Assembly with scientific annexes, vol I. New York: United Nations Scientific Committee on the Effects of Atomic Radiation; 2010.

12. Muhogora WE, Ahmed NA, Alsuwaidi JS, et al. Paediatric CT examinations in 19 developing countries: frequency and radiation dose. Radiat Prot Dosimetry. 2010;140(1):49-58.

13. Callahan MJ. CT dose reduction in practice. Pediatr Radiol. 2011;41(Suppl 2):488-92.

14. Verdun FR, Gutierrez D, Vader JP, et al. CT radiation dose in children: a survey to establish age-based diagnostic reference levels in Switzerland. Eur Radiol. 2008;18(9):1980-6.

15. Hricak H, Brenner DJ, Adelstein SJ, Frush DP, Hall EJ, Howell RW, McCollough CH, Mettler FA, Pearce MS, Suleiman OH, Thrall JH, Wagner LK. Managing radiation use in medical imaging: a multifaceted challenge. Radiology. 2011;258(3):889-905.

16. Brenner D, Elliston C, Hall E, Berdon W. Estimated risks of radiation-induced fatal cancer from pediatric CT. AJR Am J Roentgenol. 2001;176(2):289-96.

17. Brenner DJ. Radiation risks potentially associated with low-dose CT screening of adult smokers for lung cancer. Radiology. 2004; 231(2):440-5.

18. Brenner DJ, Elliston CD. Estimated radiation risks potentially associated with full-body CT screening. Radiology. 2004;232: $735-8$.

19. Brenner DJ, Shuryak I, Einstein AJ. Impact of reduced patient life expectancy on potential cancer risks from radiologic imaging. Radiology. 2011;261(1):193-8.

20. Durand DJ. A rational approach to the clinical use of cumulative effective dose estimates. AJR Am J Roentgenol. 2011;197(1): 160-2.

21. Brenner DJ, Doll R, Goodhead DT, et al. Cancer risks attributable to low doses of ionizing radiation: assessing what we really know. Proc Natl Acad Sci USA. 2003;100:13761-6.

22. • Pearce MS, Salotti JA, Little MP, et al. Radiation exposure from CT scans in childhood and subsequent risk of leukaemia and brain tumours: a retrospective cohort study. Lancet 2012; 380:499-505. The use of CT scans in children to deliver cumulative doses of about $50 \mathrm{mGy}$ might almost triple the risk of leukemia and doses of about $60 \mathrm{mGy}$ might triple the risk of brain cancer.

23. Greess H, Nömayr A, Wolf H, Baum U, Lell M, Böwing B, Kalender W, Bautz WA. Dose reduction in CT examination of children by an attenuation-based on-line modulation of tube current (CARE Dose). Eur Radiol. 2002;12(6):1571-6.

24. Papadakis AE, Perisinakis K. Angular on-line tube current modulation in multidetector CT examinations of children and adults: the influence of different scanning parameters on dose reduction. Med Phys. 2007;34(7):2864-74.

25. Perisinakis K, Papadakis AE, Damilakis J. The effect of $\mathrm{x}$-ray beam quality and geometry on radiation utilization efficiency in multidetector CT imaging. Med Phys. 2009;36(4):1258-66.

26. Sorantin E, Riccabona M, Stücklschweiger G, Guss H, Fotter R. Experience with volumetric (320 rows) pediatric CT. Eur J Radiol. 2012. doi:10.1016/j.ejrad.2011.12.001.

27. Tzedakis A, Perisinakis K, Raissaki M, Damilakis J. The effect of $\mathrm{z}$ overscanning on radiation burden of pediatric patients undergoing head CT with multidetector scanners: a Monte Carlo study. Med Phys. 2006;33(7):2472-8.

28. Christner JA, Zavaletta VA, Eusemann CD, Walz-Flannigan AI, McCollough $\mathrm{CH}$. Dose reduction in helical CT: dynamically adjustable Z-axis X-ray beam collimation. AJR Am J Roentgenol. 2010;194(1):W49-55.

29. Deak PD, Langner O, Lell M, Kalender WA. Effects of adaptive section collimation on patient radiation dose in multisection spiral CT. Radiology. 2009;252(1):140-7.

30. Lell MM, May M, Deak P, Alibek S, Kuefner M, Kuettner A, Köhler H, Achenbach S, Uder M, Radkow T. High-pitch spiral computed tomography: effect on image quality and radiation dose in pediatric chest computed tomography. Invest Radiol. 2011;46(2):116-23.

31. • Young C, Taylor AM, Owens CM. Paediatric cardiac computed tomography: a review of imaging techniques and radiation dose consideration. Eur Radiol. 2011;21(3):518-29. This provides a summary of evidenced-based recommendations with recent bibliography and in-house data discussing the various methods available for dose calculations and their inherent bias.

32. Hoang JK, Yoshizumi TT, Choudhury KR, Nguyen GB, Toncheva G, Gafton AR, Eastwood JD, Lowry C, Hurwitz LM. Organ-based dose current modulation and thyroid shields: techniques of radiation dose reduction for neck CT. AJR Am J Roentgenol. 2012;198(5):1132-8.

33. Matsubara K, Sugai M, Toyoda A, Koshida H, Sakuta K, Takata T, Koshida K, Iida H, Matsui O. Assessment of an organ-based tube current modulation in thoracic computed tomography. J Appl Clin Med Phys. 2012;13(2):3731.

34. Duan X, Wang J, Christner JA, Leng S, Grant KL, McCollough $\mathrm{CH}$. Dose reduction to anterior surfaces with organ-based tubecurrent modulation: evaluation of performance in a phantom study. AJR Am J Roentgenol. 2011;197(3):689-95.

35. Hara AK, Paden RG, Silva AC, Kujak JL, Lawder HJ, Pavlicek $\mathrm{W}$. Iterative reconstruction technique for reducing body radiation dose at CT: feasibility Study. AJR. 2009;193:764-71.

36. - Silva AC, Lawder HJ, Hara A, Kujak J, Pavlicek W: Innovations in CT dose reduction strategy: application of the adaptive statistical iterative reconstruction algorithm. AJR 2010;194: 191-199. This review shows that iterative reconstruction algorithms are a promising technique for providing diagnostic-quality CT images at significantly reduced radiation doses.

37. Marin D, Nelson RC, Schindera ST, Richard S, Youngblood RS, Yoshizumi TT, Samei E. Low-tube- voltage, high-tube-current multidetector abdominal CT: improved image quality and decreased radiation dose with adaptive statistical iterative reconstruction algorithm —initial clinical experience. Radiology. 2010; 254:145-53.

38. Pontana F, Pagniez J, Flohr T, Faivre JB, Duhamel A, Remy J, Remy-Jardin M. Chest computed tomography using iterative reconstruction vs filtered back projection (part 1): evaluation of image noise reduction in 32 patients. Eur Radiol. 2011;21(3): $627-35$.

39. Ghetti C, Ortenzia O, Serreli G. CT iterative reconstruction in image space: a phantom study. Phys Med. 2012;28(2):161-5.

40. Fleischmann D, Boas FE. Computed tomography-old ideas and new technology. Eur Radiol. 2011;21(3):510-7. 
41. Toshiba Medical. Available at http://www.toshiba-medical.eu/en/ Our-Product-Range/CT/Systems/Aquilion-16/. Accessed 20 Dec 2012.

42. Singh S, Kalra MK, Shenoy-Bhangle AS, Saini A, Gervais DA, Westra SJ, Thrall JH. Radiation dose reduction with hybrid iterative reconstruction for pediatric CT. Radiology. 2012;263(2): 537-46.

43. Miéville FA, Gudinchet F, Brunelle F, Bochud FO, Verdun FR. Iterative reconstruction methods in two different MDCT scanners: physical metrics and 4-alternative forced-choice detectability experiments-a phantom approach. Phys Med. 2012. doi:10.1016/j.ejmp.2011.12.004.

44. Miéville FA, Gudinchet F, Rizzo E, Ou P, Brunelle F, Bochud FO, Verdun FR. Paediatric cardiac CT examinations: impact of the iterative reconstruction method ASIR on image qualitypreliminary findings. Pediatr Radiol. 2011;41(9):1154-64.

45. Korn A, Fenchel M, Bender B, Danz S, Hauser TK, Ketelsen D, Flohr T, Claussen CD, Heuschmid M, Ernemann U, Brodoefel H. Iterative reconstruction in head CT: image quality of routine and low-dose protocols in comparison with standard filtered backprojection. AJNR Am J Neuroradiol. 2012;33(2):218-24.

46. McCollough $\mathrm{CH}$, Chen $\mathrm{GH}$, Kalender W, Leng S, Samei E, Taguchi K, Wang G, Yu L, Pettigrew RI. Achieving routine submillisievert CT Scanning: report from the summit on management of radiation dose in CT. Radiology. 2012;264(2):567-80.

47. Baumueller S, Winklehner A, Karlo C, Goetti R, Flohr T, Russi EW, Frauenfelder T, Alkadhi H. Low-dose CT of the lung: potential value of iterative reconstructions. Eur Radiol. 2012;22(12):2597-606.

48. Miéville FA, Berteloot L, Grandjean A, Ayestaran P, Gudinchet F, Schmidt S, Brunelle F, Bochud FO, Verdun FR. Model-based iterative reconstruction in pediatric chest CT: assessment of image quality in a prospective study of children with cystic fibrosis. Pediatr Radiol. 2012. doi:10.1007/s00247-012-2554-4.

49. International Commission on Radiological Protection. Radiological protection and safety in medicine. ICRP publication 73 . Amsterdam: Elsevier; 1997.

50. Verdun FR, Gutierrez D, Vader JP, Aroua A, Alamo-Maestre LT, Bochud F, Gudinchet F. CT radiation dose in children: a survey to establish age-based diagnostic reference levels in Switzerland. Eur Radiol. 2008;18(9):1980-6.
51. Swiss diagnostic reference level for CT. Available at: http:// www.bag.admin.ch/themen/strahlung/10463/10958/. Accessed 20 Dec 2012.

52. Dougeni E, Faulkner K, Panayiotakis G. A review of patient dose and optimisation methods in adult and paediatric CT scanning. Eur J Radiol. 2012;81(4):e665-83.

53. Image Wisely campaign. Available at. http://www.imagewisely. org/. Accessed 20 Dec 2012.

54. Image gently campaign. Available at. http://www.pedrad.org/. Accessed 20 Dec 2012.

55. Szucs-Farkas Z, Verdun FR, von Allmen G, Mini RL, Vock P. Effect of X-ray tube parameters, iodine concentration, and patient size on image quality in pulmonary computed tomography angiography: a chest-phantom-study. Invest Radiol. 2008;43(6): 374-81.

56. Yu L, Bruesewitz MR, Thomas KB, Fletcher JG, Kofler JM, McCollough $\mathrm{CH}$. Optimal tube potential for radiation dose reduction in pediatric CT: principles, clinical implementations, and pitfalls. Radiographics. 2011;31(3):835-48.

57. Verdun FR, Lepori D, Monnin P, Valley JF, Schnyder P, Gudinchet F. Management of patient dose and image noise in routine pediatric CT abdominal examinations. Eur Radiol. 2004;14(5):835-41.

58. Li J, Udayasankar UK, Toth TL, Seamans J, Small WC, Kalra MK. Automatic patient centering for MDCT: effect on radiation dose. AJR Am J Roentgenol. 2007;188(2):547-52.

59. - Geleijns J, Wang J, McCollough C. The use of breast shielding for dose reduction in pediatric CT: arguments against the proposition. Pediatr Radiol. 2010;40(11):1744-7. This presents the limitations of breast shielding when dealing with the optimization of patient dose exposure.

60. Dickerson JM, Koch BL, Adams JM, Goodfriend MA, Donnelly LF. Safety coaches in radiology: decreasing human error and minimizing patient harm. Pediatr Radiol. 2010;40(9):1545-51.

61. Schindera ST, Treier R, von Allmen G, Nauer C, Trueb PR, Vock $\mathrm{P}$, Szucs-Farkas $\mathrm{Z}$. An education and training programme for radiological institutes: impact on the reduction of the CT radiation dose. Eur Radiol. 2011;21(10):2039-45.

62. Deak PD, Smal Y, Kalender WA. Multisection CT protocols: sexand age-specific conversion factors used to determine effective dose from dose-length product. Radiology. 2010;257(1):158-66. 